\title{
Baseline bispectral index values below 46 in an octogenarian under desflurane anesthesia
}

\author{
Peter J. Lee, MD • Clint S. Wong, MD • \\ Himat Vaghadia, MBBS
}

Received: 13 May 2010/Accepted: 10 June 2010/Published online: 24 July 2010

(C) Canadian Anesthesiologists' Society 2010

\section{To the Editor:}

The Bispectral Index ${ }^{\mathrm{TM}}$ (Aspect Medical Systems, Newton, MA, USA), a set of variables ranging from 0 to 100 that correlate with the degree of patient sedation, has been used to monitor the depth of anesthesia. Values $<60$ correlate with a small probability of consciousness ${ }^{1}$ and a bispectral index (BIS) value in the range of 40 to 60 has been recommended for adequate depth of anesthesia. ${ }^{\mathrm{A}}$ Dementia may be associated with an increase in slow-wave and a decrease in fast-wave electroencephalogram activity that leads to a reduction of the BIS baseline in the awake state. $^{2}$ Bispectral index values are significantly lower in geriatric patients with Alzheimer's disease or vascular dementia than they are in geriatric patients without these conditions. ${ }^{3}$ In a nonagenarian, emergence from propofol anesthesia has been described at a BIS value of $52 .{ }^{4}$ Therefore, it has been suggested that all elderly patients undergoing BIS-monitored anesthesia should have a baseline recording prior to induction of anesthesia to alert the anesthesia provider to the possibility of low initial values. ${ }^{3}$

We describe a case of a patient undergoing laparoscopic cholecystectomy under general anesthesia with desflurane wherein both pre-induction consciousness and post-emergence extubation consciousness correlated with a BIS value generally associated with adequate depth of anesthesia.

An 88-yr-old female who was American Society of Anesthesiologists physical status III with mild cognitive impairment, depression, and renal insufficiency, was admitted for a laparoscopic cholecystectomy with a diagnosis of acute cholecystitis. The patient's regular medication

P. J. Lee, MD $(\bowtie) \cdot$ C. S. Wong, MD · H. Vaghadia, MBBS

Department of Anesthesia, Pharmacology and Therapeutics,

University of British Columbia, Vancouver, BC, Canada

e-mail: peterjohnlee@hotmail.com consisted of citalopram, a selective serotonin reuptake inhibitor, and a combination of diclofenac and misoprostol. In addition to routine monitors, a BIS monitor sensor $\left(\mathrm{A}-2000^{\mathrm{TM}}\right.$ XP Bispectral Index ${ }^{\mathrm{TM}}$ Monitoring System, software version 3.30, Aspect Medical Systems, Newton, MA, USA) was applied to the patient's forehead and left temporal area. This stand-alone unit computes BIS, 95\% spectral edge frequency, suppression ratio, and signal quality index. It uses an epoch duration of two seconds and an update rate of one second for the BIS monitoring. Signal quality index values for the BIS monitor ranged from 80 to 85 throughout the case. A BIS of 46 was observed in the patient's awake state. Anesthesia was induced with propofol $60 \mathrm{mg}$ and rocuronium $30 \mathrm{mg}$ and was maintained with 3.5-5.4\% desflurane in oxygen and air (1:2) and a mixed infusion of fentanyl $5 \mu \mathrm{g} \cdot \mathrm{mL}^{-1}$ and hydromorphone $0.05 \mathrm{mg} \cdot \mathrm{mL}^{-1}$. Total narcotic administration during the procedure was fentanyl $180 \mu \mathrm{g} i v$ and hydromorphone $1.5 \mathrm{mg} i v$. Intraoperative BIS values ranged from 31-39. The 3.5-hr surgical course was uneventful, and the patient returned to spontaneous ventilation toward the end of the case. At the time of tracheal extubation, the patient was alert, awake, and spontaneously breathing, and the BIS value was 92. Shortly thereafter, while she was still in the operating room in an alert and awake state and breathing spontaneously, her BIS value was 45 . The patient was observed in the postanesthetic recovery room without event and she was discharged one hour later.

Given that the algorithm for the BIS was derived from a database of predominantly fit, healthy adult patients, this case highlights the fact that the elderly patient with cognitive impairment or dementia may have a lower than

\footnotetext{
A BIS Clinical Reference Manual. Newton, Massachusetts: Aspect Medical Systems, Inc., 1998.
} 
expected BIS value. As a result, it is unclear whether the recommended target BIS value for adequate hypnosis in the general population (40-60) is adequate for the elderly patient with cognitive impairment. Until correlation of BIS values to clinical endpoints such as loss of consciousness and lack of both implicit and explicit recall can be determined in demented and non-demented patients and elderly and non-elderly patients, routine use of BIS in this population may not ensure detection of unintended awareness under general anesthesia.

Competing interests None declared.

Specifically, none of the authors has any financial interest related to patents and/or share holdings in corporations involved in the development and/or marketing of the BIS monitor.

\section{References}

1. Glass PS, Bloom M, Kearse L, Rosow C, Sebel P, Manberg P. Bispectral analysis measures sedation and memory effects of propofol, midazolam, isoflurane, and alfentanil in healthy volunteers. Anesthesiology 1997; 86: 836-47.

2. Renna $M$, Venturi $R$. Bispectral index and anaesthesia in the elderly. Minerva Anesthesiol 2000; 66: 398-402.

3. Renna M, Handy J, Shah A. Low baseline bispectral index of the electroencephalogram in patients with dementia. Anesth Analg 2003; 96: 1380-5.

4. Kakinohana M, Nakamura S, Miyata $Y$, Sugahara K. Emergence from propofol anesthesia in a nonagenarian at a bispectral index of 52. Anesth Analg 2005; 101: 169-70. 